\section{Afalia congénita: reporte de un caso y revisión de la bibliografía; de la patogenia a la reconstrucción fálica}

Carrillo-Córdova LD, ${ }^{1}$ Carrillo-Esper, ${ }^{2}$ Carrillo-Córdova JR, ${ }^{3}$ González-Mondragón $\mathrm{MB},{ }^{4}$ Mosqueira-Mondragón $\mathrm{CF}^{4}$

Resumen

ANTECEDENTES: la afalia congénita es una enfermedad excepcional, asociada con malformaciones musculoesqueléticas y cardiovasculares.

CASO CLÍNICO: recién nacido con diagnóstico de afalia congénita, pretérmino de 33.4 semanas de gestación, adecuado peso para la edad gestacional, con un soplo sistólico multifocal, con ausencia de pene y uretra, ano imperforado, fístula uretrocutánea perianal, con gasto de orina clara a través de ésta. Al segundo día de nacido se realizó una colostomía, fístula mucosa y se colocó un catéter venoso central. Posteriormente se comentó con los padres la necesidad de una cistotomía, que fue rechazada.

DISCUSIÓN: la limitación más importante al momento de la reconstrucción del pene es que no existe un sustituto para el tejido eréctil; por lo tanto, el neo-falo debe de ser satisfactorio en el aspecto estético, funcional y psicológico.

PALABRAS CLAVE: afalia, reconstrucción de pene, malformación genitourinaria.

Rev Mex Urol. 2017 January;77(1):71-78.

\section{Congenital aphallia: A case report and a literature review from pathogenesis to phallic reconstruction}

Carrillo-Córdova LD, ${ }^{1}$ Carrillo-Esper, ${ }^{2}$ Carrillo-Córdova JR, ${ }^{3}$ González-Mondragón $\mathrm{MB},{ }^{4}$ Mosqueira-Mondragón $\mathrm{CF}^{4}$

\section{Abstract \\ BACKGROUND: Congenital aphallia is a rare disease associated with musculoskeletal and cardiovascular malformations.}

CLINICAL CASE: A pre-term (33.4 weeks of gestation) neonate was diagnosed with congenital aphallia. The patient's weight was adequate in relation to gestational age. The infant presented with a multifocal systolic murmur and absence of the penis and urethra, as well as an imperforate anus and perianal urethrocutaneous fistula that was the

\begin{abstract}
${ }^{1}$ Departamento de Urología, Hospital General de México Dr. Eduardo Liceaga, Ciudad de México, México. ${ }^{2}$ Áreas críticas CENIAQ, Instituto Nacional de Rehabilitación, Ciudad de México, México.

${ }^{3}$ Departamento de Cirugía Plástica y Reconstructiva, Hospital General Dr. Manuel Gea González, Ciudad de México, México.

${ }^{4}$ Departamento de Cirugía Pediátrica, Hospital General de México Dr. Eduardo Liceaga, Ciudad de México, México.
\end{abstract}

Recibido: octubre 2016

Aceptado: enero 2017

Correspondencia

Dr. Jorge Carrillo Córdova

dr.carrillo.plastica@gmail.com

Este artículo debe citarse como

Carrillo-Córdova LD, Carrillo-Esper, Carrillo-Córdova JR, González-Mondragón MB, Mosqueira-Mondragón CF. Afalia congénita: reporte de un caso y revisión de la bibliografía; de la patogenia a la reconstrucción fálica. Rev Mex Urol. 2017 ene;77(1):71-78. 
exit route for clear urine output. Colostomy and mucosal fistula were performed on the second day of birth and a central venous catheter was placed. The need for cystostomy was later discussed with the parents, but was rejected.

DISCUSSION: The most important limitation at the time of penile reconstruction is that there is no substitute for erectile tissue. Therefore, the neo-phallus must be satisfactory from the aesthetic, functional, and psychologic perspectives.

KEY WORDS: Aphallia; Penile reconstruction; Genitourinary malformation
Correspondence

Dr. Jorge Carrillo Córdova

dr.carrillo.plastica@gmail.com

\section{ANTECEDENTES}

La afalia congénita, o agenesia de pene, es una enfermedad congénita infrecuente, con consecuencias funcionales, estéticas y psicológicas devastadoras. ${ }^{1}$ La prevalencia mundial es de un caso por cada 10 a 30 millones. ${ }^{2-5}$ La mayoría de los pacientes tiene cariotipo $46 \mathrm{XY}$.

La fisiopatogenia de esta enfermedad se asocia con alteraciones en la formación del tubérculo genital, que a su vez se vincula con malformaciones genitourinarias y sistémicas. ${ }^{5-7}$ En los pacientes con afalia congénita suelen coexistir malformaciones renales, cardiacas y musculoesqueléticas. Éstas se explican debido a que el sistema digestivo y la vía urinaria caudal embrionarios tienen un origen en común: la cloaca; un hallazgo habitual en estos pacientes son las fístulas uretrorrectales. En este caso, al existir un ano imperforado, el trayecto fistuloso de la uretra se dirige al área perianal.

\section{Comunicación del caso}

Recién nacido en el Hospital General de México, con diagnóstico de afalia congénita, pretérmino de 33.4 semanas de gestación; madre sana, con antecedente de un embarazo, nacido por cesárea debida a trabajo de parto prematuro. Ambos padres estaban sanos, sin antecedentes familiares de enfermedades congénitas. El ultrasonido fetal inicial, a las 22 semanas, mostró un feto femenino, sin algún otro diagnóstico prenatal.

A la exploración física se encontró un recién nacido con adecuado peso para la edad gestacional, con un soplo sistólico multifocal, con ausencia de pene y uretra. Ambos testículos estaban descendidos dentro de la bolsa escrotal, de características normales y rafe medio normal. Ano imperforado, con fístula uretrocutánea perianal, con gasto de orina clara a través de ésta. Además, una malformación en el pie izquierdo, con fusión digital del segundo y tercer dedo. Figura 1

Después de haber estabilizado al paciente se obtuvo el cariotipo: cromosomas XY. El ultrasonido abdominal reportó: vejiga pequeña, uretra con trayecto fistuloso perianal y el riñón derecho en el hueco pélvico y el izquierdo en la cavidad abdominal (Figura 2). La ecografía cardiaca reportó persistencia del conducto arterioso, insuficiencia tricuspídea y atrial y crecimiento biventricular con función conservada.

La función renal se complementó con un gamagrama renal (MAG 3-furosemida) que mostró al 


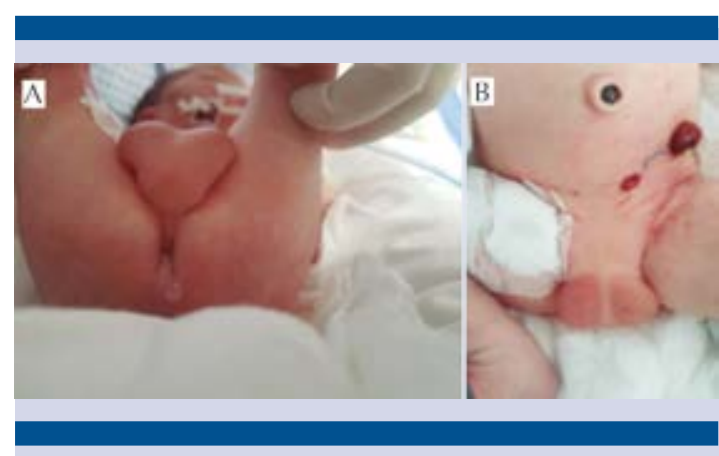

Figura 1. Bolsa escrotal íntegra con testículos en su interior, ano perforado con fístula uretrocutánea perianal (A), afalia con presencia de derivación intestinal (B).

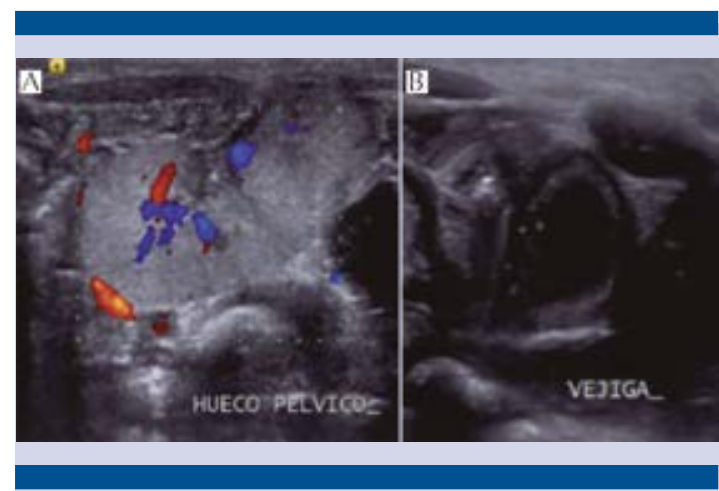

Figura 2. Riñón en el hueco pélvico con adecuado flujo Doppler (A), cavidad vesical con pared engrosada y conducto uretral (B).

riñón derecho abdominal, con aclaramiento de $11.9 \mathrm{~mL} / \mathrm{min}$ y el izquierdo en el hueco pélvico, con depuración de $2.2 \mathrm{~mL} / \mathrm{min}$; no se evidenciaron los uréteres y la vejiga se encontró en el hueco pélvico. (Figura 3)

Al segundo día de nacido se realizó una colostomía, fístula mucosa y se colocó un catéter venoso central. Posteriormente se comentó con los padres la necesidad de una cistostomía, que fue rechazada. En la actualidad el paciente permanece en vigilancia en la consulta externa de Urología.

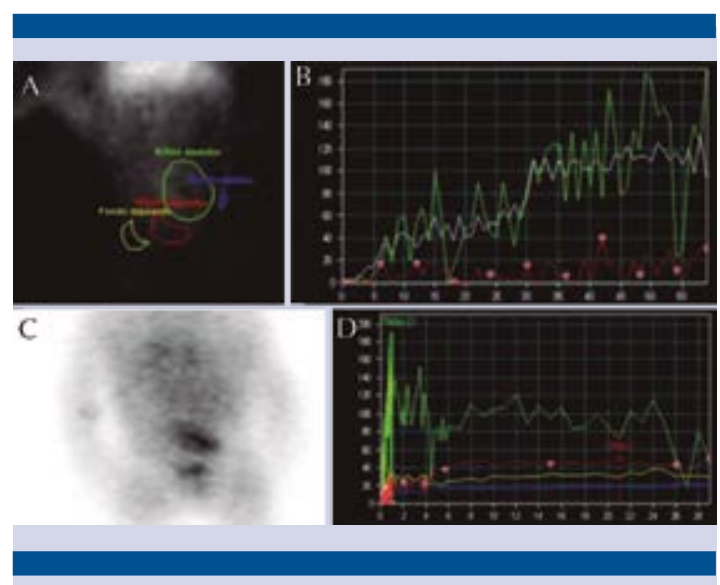

Figura 3. Gamagrama renal con MAG 3. Imagen lateral en fase de eliminación, donde se evidencia en verde el riñón derecho abdominal, en rojo el riñón izquierdo y en amarillo la vejiga (A), la gráfica de flujo (B), una toma anterior de la fase de perfusión $(\mathbf{C})$, y la gráfica renográfica $(\mathrm{D})$.

\section{DISCUSIÓN}

La afalia es una malformación genitourinaria de la que en todo el mundo se han reportado menos de 100 casos. En la bibliografía hay casos esporádicos, pues la baja prevalencia no ha estimulado el interés para estudiarla. Se desconoce el mecanismo de la falla en el desarrollo del tubérculo genitourinario; en la mayoría de los casos el cariotipo es XY, con valores de hormonas sexuales en parámetros normales, con el eje hipotálamo gonadal intacto.

\section{Fisiopatogenia}

El tubérculo gonadal se forma entre la cuarta y quinta semanas de vida intrauterina, posterior a la proliferación mesenquimatosa de la eminencia cloacal. La proliferación mesenquimatosa anormal da como resultado malformaciones genitourinarias, entre ellas la afalia congénita. ${ }^{8}$

Está demostrado que las alteraciones tempranas en el desarrollo del embrión afectan la eminencia 
cloacal, originan comunicaciones uretrorrectales proximales y anormalidades genitourinarias. Las alteraciones tardías en el desarrollo resultan en fístulas uretrocutáneas abiertas de manera perianal, sin otras alteraciones urogenitales. En estos pacientes el escroto y los testículos son de características normales. ${ }^{9,10}$

La afalia es una malformación congénita, frecuentemente asociada con otras malformaciones urogenitales y sistémicas. En algunas series se ha reportado una asociación tan alta como $50 \%$ con otras malformaciones congénitas urogenitales: criptorquidia, agenesia renal, displasia, riñón poliquístico, riñón en herradura, agenesia prostática y de la vejiga, hidronefrosis, reflujo vesicoureteral, agenesia ureteral, hidrouréter, fístulas uretrorrectales y vesicorrectales, vejiga poliquística y riñón ectópico pélvico. 5,7,11-20

Está descrita la asociación con otras malformaciones congénitas, como: siringomielia, espina bífida, asociación VATER, hepatomegalia, páncreas anular, insuficiencia tricuspídea, persistencia del conducto arterioso, anormalidades gastrointestinales, síndrome de abdomen de ciruela, síndrome de Potter, síndrome de Treacher Collins, retraso mental, ano imperforado, defectos musculoesqueléticos y pie equino varo. $5,7,12,17-20$

\section{Diagnóstico}

La ausencia de pene con escroto adecuadamente desarrollado, con ano imperforado y fístula uretrocutánea perianal son hallazgos comunes. Es necesario saber diferenciar entre pene oculto, pene rudimentario, micropene, epispadias, hipospadias, amputación intrauterina del pene y pseudohermafroditismo. ${ }^{21}$

El diagnóstico se establece luego de la adecuada evaluación clínica; es necesario establecer el cariotipo y realizar estudios radiológicos de extensión, como el ultrasonido, resonancia magnética, cistografía y gamagrama renal. Estos son de gran ayuda en la evaluación completa de la vía urinaria y para descartar otras malformaciones congénitas asociadas con esta afección y así guiar el tratamiento. 22,23

\section{Clasificación}

Con base en la posición de la uretra, en relación con el esfínter anal, se propuso una clasificación de la afalia que agrupa a los pacientes en tres variables: postesfinterianas, preesfinterianas y atresia uretral. La posición más proximal de la uretra, en relación con el esfínter anal, resulta en mayor cantidad de malformaciones asociadas y en mortalidad más elevada. ${ }^{24}$ Otra clasificación es la que se fundamenta en la coexistencia de otras malformaciones congénitas, que implica peor pronóstico y mayor mortalidad. ${ }^{12}$

\section{Tratamiento}

Uno de los aspectos que más ha cambiado, con respecto al tratamiento de pacientes con afalia, es la conducta a tomar en cuanto al fenotipo buscado. El tratamiento solía consistir en la reasignación de género; hasta finales del decenio de 1990 la conducta más habitual era la orquiectomía bilateral, con reconstrucción vaginal. La orquiectomía se recomendaba en una etapa temprana, para prevenir la impronta masculina en el cerebro neonatal, secundaria a la producción de testosterona que se inicia entre el décimo y sexagésimo días de vida extrauterina. ${ }^{25-29}$

Meyer-Bahlburg y colaboradores estudiaron, prospectivamente, pacientes con cariotipo XY con agenesia de pene, ablación de éste y extrofia de cloaca, que se criaron como varones y pacientes con cariotipo $X Y$ asignados al fenotipo femenino y se criaron como mujeres. Encontró que 100\% de los criados como varones, permanecieron varones $(n=311)$, y de los que se criaron como mujeres, 
$22 \%$ cambiaron de género y $13 \%$ sufrieron disforia de género; esto comparado con el cambio de género de la población general de 1:10,000 en hombres y 1:30,000 en mujeres. ${ }^{30}$

Reiner y su grupo estudiaron la viabilidad de asignar un fenotipo femenino a hombres nacidos $X Y$. Compararon la identidad sexual declarada y encontraron que de 60 pacientes $53 \%$ declararon una identidad sexual masculina $y$, específicamente de pacientes con afalia criados y reconstruidos sexualmente como mujeres, 50\% refirieron identidad sexual masculina. El 100\% de los pacientes criados como hombres refirieron identidad sexual masculina, desde edades tan tempranas como los cuatro años. ${ }^{31}$

El tratamiento de la afalia debe ser multidisciplinario (pediatras, genetistas, cirujanos pediatras, urólogos, cirujanos plásticos, psiquiatras y especialistas dirigidos a tratar las otras malformaciones sistémicas asociadas) desde el nacimiento hasta la vida adulta. ${ }^{19}$

De Castro fue el pionero de los cambios en la atención de pacientes con afalia congénita. Para prevenir problemas de identidad sexual él propuso no realizar el cambio fenotípico de género y criar al niño según su cariotipo y producción hormonal. Otra de sus recomendaciones fue la plastia temporal de pene, a edad temprana, para ayudar al niño a formar una salud sexual lo más normal posible, hasta poder llevar a cabo una plastia de pene definitiva en la pubertad. Posteriormente se describió que la reconstrucción durante la infancia causa mayor dificultad técnica para la reconstrucción definitiva. ${ }^{32,33}$

La recomendación es tratar a los pacientes con afalia en tres fases: aguda, subaguda y crónica. El neonato con afalia requiere el establecimiento de su cariotipo para descartar otras afecciones susceptibles de corrección, como la hiperplasia adrenal. En la fase aguda debe evaluarse minu- ciosamente la anatomía de la vía urinaria para detectar alguna obstrucción y derivarla. Los pacientes con esta afección son propensos a las infecciones urinarias, sepsis y desequilibrios hidroelectrolíticos, como la acidosis metabólica hiperclorémica. Cuando lo anterior se identifique debe actuarse lo más pronto posible. ${ }^{19}$ En caso de sepsis u obstrucción de la vía urinaria lo indicado es la cistostomía, que ayudará a drenar de la vía urinaria el foco infeccioso.

En la fase subaguda la conducta médica se fundamenta en la necesidad de los pacientes de tener una vía de drenaje vesical permanente. En caso de agenesia uretral o vejiga pequeña se deja una cistostomía a permanencia. En los pacientes con uretra debe formarse un meato perineal, o dejar una cistostomía; siempre separando la uretra de cualquier fístula que pudiera existir (uretrorectal, uretro-cutánea perianal).

Para lo anterior es necesario un estudio de extensión que permita conocer con precisión toda la anatomía urinaria (resonancia magnética), identificar el cuello vesical, la próstata y la vía uretral. Durante esta etapa puede valorarse la conformación de un pseudopene que ayude al desarrollo psicológico del paciente.

La fase crónica es la de mayores retos para el equipo médico y tiene como objetivo la realización de un neofalo, estética y funcionalmente adecuado, que ayude al paciente a tener una vida plena. Algunos autores han recomendado que la reconstrucción para ayudar al óptimo desarrollo psicosexual del paciente y reducir el tamaño de la cicatriz se lleve a cabo durante la pubertad (10 a 15 años). ${ }^{32}$

\section{Tratamiento reconstructivo en pacientes con afalia congénita}

La bibliografía enfocada a la reconstrucción de pacientes con afalia congénita es poca, la mayor 
parte de la información existente para valorar el tratamiento reconstructivo de esta enfermedad proviene de artículos que exponen opciones reconstructivas en pacientes con traumatismos, resecciones oncológicas o reasignación de género.

Al principio, la reconstrucción de los pacientes con afalia congénita se encaminó hacia la reasignación de género; es decir, a la feminización del individuo: orquiectomía, vaginoplastia, reconstrucción labial, entre otras. Por desgracia, estos pacientes crecían y presentaban disforia de género, que a su vez originaba grandes problemas en la vida adulta. Por esto la conducta actual se dirige a criar a estos pacientes como varones, hacerles una reconstrucción peneana de acuerdo con su edad.

Uno de los aspectos relevantes es el momento idoneo para la reconstrucción. Puesto que la mayor parte de los procedimientos para la reconstrucción total del pene son complejos e implican múltiples cirugías, la pubertad se considera el límite para la intervención quirúrgica, posterior a una evaluación paidopsiquiátrica correcta. $^{34}$

Castro y colaboradores reportan, en cuatro casos, la aplicación de un colgajo preliminar antes de la pubertad, con buenos resultados a corto plazo y la necesidad de un colgajo definitivo años más tarde, cuando el cuerpo haya alcanzado su crecimiento máximo. Además, reportan considerables complicaciones urológicas y formación de cicatrización excesiva, los autores concluyen no operar a los pacientes antes de los 15 años. ${ }^{32}$

El tema de controversia es que a edad temprana la cirugía se asocia con mayor número de procedimientos, circunstancia que incrementa la morbilidad; por ello la reconstrucción en un solo tiempo es el tratamiento de elección, cuando ya han alcanzado la madurez sexual (pubertad). En
Cuadro 1. Malformaciones congénitas asociadas a la afalia

\begin{tabular}{|c|c|}
\hline $\begin{array}{l}\text { Malformaciones } \\
\text { urogenitales }\end{array}$ & $\begin{array}{l}\text { Malformaciones } \\
\text { sistémicas }\end{array}$ \\
\hline Criptorquidia & Siringomielia \\
\hline Agenesia renal & Espina bífida \\
\hline Hipoplasia renal & Páncreas anular \\
\hline Ectopia renal & Hepatomegalia \\
\hline Riñón poliquístico & Asociación VATER \\
\hline Riñón en herradura & Insuficiencia tricuspídea \\
\hline Hidronefrosis & $\begin{array}{l}\text { Persistencia de conducto } \\
\text { arterioso }\end{array}$ \\
\hline Agenesia de próstata & $\begin{array}{l}\text { Anormalidades } \\
\text { gastrointestinales }\end{array}$ \\
\hline Agenesia vesical & $\begin{array}{l}\text { Síndrome de abdomen en } \\
\text { ciruela }\end{array}$ \\
\hline Quiste vesical & Síndrome de Potter \\
\hline Reflujo vesico ureteral & Síndrome de Treacher Collins \\
\hline Hidrouréter & Retraso mental \\
\hline Agenesia ureteral & Ano imperforado \\
\hline Fístulas uretrales & $\begin{array}{c}\text { Defectos } \\
\text { musculoesqueléticos }\end{array}$ \\
\hline
\end{tabular}

estos casos la reconstrucción del pene se basa en la información obtenida de pacientes con lesiones traumáticas, en quienes la reconstrucción se efectúa, en la mayoría de los casos, con colgajos libres.

La limitación más importante, al momento de la reconstrucción del pene, es la falta de un sustituto para el tejido eréctil. El neofalo debe ser estéticamente satisfactorio, funcional y aceptable desde el punto de vista psicológico. Los primeros procedimientos encaminados a esta tarea se basaban en colgajos locales, que fallaban y tenían como consecuencias: fístulas, estenosis y estructuras mal delimitadas.

Con la introducción de la microcirugía, la faloplastia en un solo tiempo se ha convertido en el procedimiento de elección. La primera 
opción es el colgajo radial de antebrazo. Las alternativas reconstructivas de estos pacientes debe analizarlas cuidadosamente un equipo multidisciplinario. En los últimos años se han descrito modificaciones a este colgajo y se han propuesto nuevos colgajos con mejor estética, que facilitan la recuperación psicofuncional. ${ }^{35}$

La reconstrucción peneana se efectúa dependiendo de las porciones afectadas; de manera general se utilizan los siguientes procedimientos: 36,37

1. Cuerpo del pene. Se forma mediante colgajos libres, casi todos ellos fasciocutáneos e, incluso, en algunos osteo fasciocutáneos. Destacan el colgajo radial, escapular y de peroné. ${ }^{38}$

2. Reconstrucción del glande. La corona y el glande pueden reconstruirse mediante injertos cutáneos.

3. Escroto. Para la reconstrucción escrotal pueden utilizarse colgajos locales $y$, en algunos casos, reconstrucción en un segundo tiempo, posterior a la expansión de tejido circundante.

4. Testículos. Mediante prótesis que pueden diseñarse dependiendo de la edad, género y características del paciente.

\section{CONCLUSIÓN}

La afalia congénita se asocia con otras malformaciones genitourinarias y sistémicas. Su repercusión no solo es en el paciente y la familia, sino también en el ámbito psicosocial, con pronóstico reservado. El tratamiento de estos pacientes debe ser multidisciplinario, por la complejidad de sus manifestaciones. En la actualidad hay unanimidad en cuanto a la atención médica fundamentada en el cariotipo y los valores hormonales, que deben atenderse como varones y promover la reconstrucción fálica para un mejor desarrollo psicosocial. La recomendación actual de tratamiento en la fase aguda se enfoca en los desequilibrios hidroelectrolíticos e infecciosos, y en garantizar el adecuado drenaje de la vía urinaria para prevenir complicaciones. La reconstrucción fálica tardía es parte de la atención integral de los pacientes.

\section{REFERENCIAS}

1. Kane A, Ngom G, Ndour O, Alumeti D. Aphallia: A case report and literature review. Afr J Paediatr Surg 2011;8:324.

2. Saeid Aslanabadi M, Zarrintan S, Habib Abdollahi M, Reza Rikhtegar M, Samad Beheshtirouy M, Davoud Badebarin M. A Rare Case of Aphallia with Right Kidney Hypoplasia and Left Kidney Dysplasia. Arch Iran Med 2015;18:257.

3. Mane SB, Thakur A, Dhende NP, Obaidah A, Acharya H. Single-stage feminizing genitoplasty in aphallia through an anterior saggital approach. J Pediatr Surg 2009;44:22332235.

4. Shamsa A, Kajbafzadeh A, Javad Parizadeh S, Zare MA, Abolbashari M. Aphallia associated with urethro- rectal fistula and stones in the bladder and urethra. Saudi J Kid Dis Transplant 2008;19:435.

5. Wang H, Guo K, Wang J, Liu L, Li F. Aphallia in an adult male with 46, XY karyotype. Int J Urol 2011; 18: 540-542.

6. Rattan KN, Kajal P, Pathak M, Kadian YS, Gupta R. Aphallia: experience with 3 cases. J Pediatr Surg 2010; 45: e13-e16.

7. Gredler ML, Seifert AW, Cohn MJ. Morphogenesis and Patterning of the Phallus and Cloaca in the American Alligator, Alligator mississippiensis. Sex Dev 2014;9:53-67.

8. Santana-Rios Z, Fernandez-Noyola G, Cantellano-Orozco $\mathrm{M}$, et al. Congenital aphallia: management with urethral advancement. Rev Mex Urol. 2011;71:239-243.

9. Choudhury SR, Maji BP. Penile agenesis: an unusual variant. Int Urol Nephrol 1993;25(1):71-6 [Review].

10. Di Benedetto V, Idotta R, Lebet $M$, et al. Penis, bladder and urethral agenesis associated with anorectal malformation in a living male neonate. Case report. Clin Exp Obstet Gynecol 1999;26(3-4):225-6.

11. Kessler WO, McLaughlin III AP. Agenesis of penis. Embryology and management. Urology 1973;1(3):226-9.

12. Evans JA, Erdile LB, Greenberg CR, et al. Agenesis of the penis: patterns of associated malformations. Am J Med Genet 1999;84(1): 47-55.

13. Dusmet $M$, Fête $F$, Crusi A, et al. VATER association: report of a case with three unreported malformations. J Med Genet 1988;25(1):57-60.

14. Roth Jr JK, Marshall RH, Angel JR, et al. Congenital absence of penis. Urology 1981;17(6):579-83. 
15. Kuga T, Esato K, Sase M, et al. Prune-belly syndrome with penile and urethral agenesis: report of a case. J Pediatr Surg 1998;33(12):1825-8.

16. Arai $M$, Suzuki S, Ishino $H$, et al. Penile agenesis complicated by Potter sequence. Arch Gynecol Obstet 2001;265(4):219-20.

17. Rennert WP. Penile agenesis associated with Treacher Collins syndrome. S Afr Med J 2002;92(5):347-9 [Review].

18. Kamal N, Rattan P, Kajal MP, et al. Aphallia: Experience with 3 cases. J Pediatr Surg 2010;45(1):E13-6.

19. Aditya Joshi, Jody Gross, Thomalla JV. Congenital Aphallia: Review of Pathogenesis and Current Treatment Guidelines. Urology 2015;86:384e387.

20. Fereshteh Talebpour Amiri, Davood Nasiry Zarrin Ghabaee, Ramezan Ali Naeimi M.Sc., Seyed Javad Seyedi, Seyed Abdollah Mousavi. Aphallia: Report of three cases and literature review. Int J Reprod BioMed 2016;4(4):279-284.

21. SoderdahI DW, Brosman SA, Goodwin WE. Penile agenesis. J Urol 1972;108(3):496-9.

22. Lapointe SP, Wei DC, Hricak $\mathrm{H}$, et al. Magnetic resonance imaging in the evaluation of congenital anomalies of the external genitalia. Urology 2001 Sep;58(3):452-6.

23. Coquet-Reinier B, Merrot T, Chaumoître K, et al. Atypical aphallia. Pediatr Surg Int 2007;23(11):1131-3.

24. Skoog SJ, Belman AB. Aphallia: its classification and management. J Urol 1989;141(3):589-92.

25. Oesch IL, Pinter A, Ransley PG. Penile agenesis: a report of six cases. J Pediatr Surg 1987 Feb;22(2):172-4.

26. [Hendren WH. The genetic male with absent penis and urethrorectal communication: experience with 5 patients. J Urol 1997;157(4):1469-74.

27. Bruch SW, Meuli M, Harrison MR. Immediate reconstruction for penile agenesis. J Pediatr Surg 1996;31(8):1152-3 [discussion 1154].
28. Shah AA, Shah AV. Neonatal genital reconstruction in penile agenesis by anterior sagittal anorectovaginourethroplasty. Pediatr Surg Int 2003;19(9-10):689-92.

29. Glüer S, Fuchs J, Mildenberger H. Diagnosis and current management of penile agenesis. J Pediatr Surg 1998;33(4):628-31.

30. Meyer-Bahlburg HF. Gender identity outcome in female raised $46, X Y$ persons with penile agenesis, cloacal exstrophy of the bladder, or penile ablation. Arch Sex Behav 2005;34:423-438.

31. Reiner WG. Gender identity and sex-of-rearing in children with disorders of sexual differentiation. J Pediatr Endocrinol Metab 2005;18(6):549-53.

32. De Castro R, Merlini E, Rigamonti W, et al. Phalloplasty and urethroplasty in children with penile agenesis: preliminary report. J Urol 2007;177(3):1112-6.

33. Perovic SV, Djinovic R, Bumbasirevic M, et al. Total phalloplasty using a musculocutaneous latissimus dorsi flap. BJU Int 2007;100(4): 899-905.

34. Selvaggi G, Elander A. Penile reconstruction. Curr Opin Urol 2008 Nov;18(6):589-97.

35. Kim S, Lee K, Kwon Y, Cha B. Phalloplasty using radial forearm osteocutaneous free flaps in female-to-male transsexuals. Br J Plast Surg [Internet] 2016;62(3):30917.

36. Palminteri E, Berdondini E, Lazzeri M, Mirri F, Barbagli G, Patologica A, et al. Resurfacing and Reconstruction of the Glans Penis. Eur Urol 2007;52(3):893-900.

37. Salgado CJ, Monstrey S. Reconstruction of the Penis After Surgery. Urol Clin NA [Internet]. 37(3):379-401.

38. Wang H, Li SK, Yang MY, Li YQ, Li Q, Chen W, et al. A free scapular skin flap for penile reconstruction. J Plast Reconstr Aesthet Surg 2007;60(11):1200-3.

\section{AVISO PARA LOS AUTORES}

Revista Mexicana de Urología tiene una nueva plataforma de gestión para envío de artículos. En: https://www.revisionporpares.com/index.php/RMUrol podrá inscribirse en nuestra base de datos administrada por el sistema Open Journal System (OJS) que ofrece las siguientes ventajas para los autores:

- Subir sus artículos directamente al sistema.

- Conocer, en cualquier momento, el estado de los artículos enviados, es decir, si ya fueron asignados a un revisor, aceptados con o sin cambios, o rechazados.

- Participar en el proceso editorial corrigiendo y modificando sus artículos hasta su aceptación final. 